\title{
Evolution of Wire Antennas in Three Dimensions using a Novel Growth Process
}

\author{
Mark Dorica and Dennis Giannacopoulos \\ Department of Electrical and Computer Engineering, McGill University \\ 3480 University Street, Montréal, Québec, Canada \\ dennis.giannacopoulos@ mcgill.ca
}

\begin{abstract}
Exploring the vast search space of possible wire antenna designs is a task well-suited for genetic algorithms. Many of the current implementations use a direct mapping between genome and antenna feature. We explore the evolution of antennas using a growth process. The design of wire antennas for communication applications demonstrates the capabilities of this approach.
\end{abstract}

\section{INTRODUCTION}

Communication, radar, and remote sensing applications all require a multitude of different types of wire antennas. Designing these antennas by hand, or by local optimization methods is time consuming, costly, and requires expertise. To address this situation, evolutionary algorithms have been developed for wire antenna design [1]-[2].

One common feature of most previous approaches is that they employ a direct mapping between genotype and phenotype. In other words, each segment in the design is directly represented within the genome. As an alternative, we propose an indirect mapping scheme, in which the genome encodes an agent. The antenna then undergoes an unconstrained growth phase, where each growth step is governed by the decisions of the agent. On some level, this is analogous to growth phases that occur in nature, where development is determined primarily by genetics. Such indirect mappings are largely unexplored in engineering design and are in line with the bio-inspired concept of using compact representations for complex structures. One such agent architecture is briefly described below.

\section{NEW AgENT FOR CONTROL OF ANTENNA GROWTH}

The agent architecture consists of 3 fuzzy inference systems (FIS), which are specified by the genome. Each FIS has 3 inputs and a single output. There are 3 membership functions (MFs) for each input and output of the FIS. Encoded within the genome are 27 genes for each of the possible rules. The center and width of each Gaussian MF is also specified within the genome. For a given input or output, all MFs have the same width. The number of antenna growth iterations can also be genetically controlled.

In basic terms, the current cell (antenna node) state is the input to each FIS. The next state is a combination of the average of the current state and the FIS outputs. The growth direction is derived from the three components of the cell state. Cell growth then proceeds in parallel for cells having a high enough affinity for growth.

\section{APPlication to Wire ANTENNA DESIGN}

The design space is $15 \times 15 \times 15 \mathrm{~cm}$. Each segment length is approximately $1 \mathrm{~cm}$. The design goal is a $1.9 \mathrm{GHz}$ antenna with uniform right hand circular polarization (RHCP) coverage, a low voltage standing wave ratio (VSWR), and low crosspolarization. These are essentially the targets specified for modern communication applications [2]. The genetic algorithm (GA) is steady-state, with replacement set to 0.4 and a population of 200. Crossover and mutation probabilities are set to 0.7 , and 0.05 respectively. The GA is run for 100 generations. One can put bounds on the number of growth iterations to limit the size and increase the practicality of the final design.

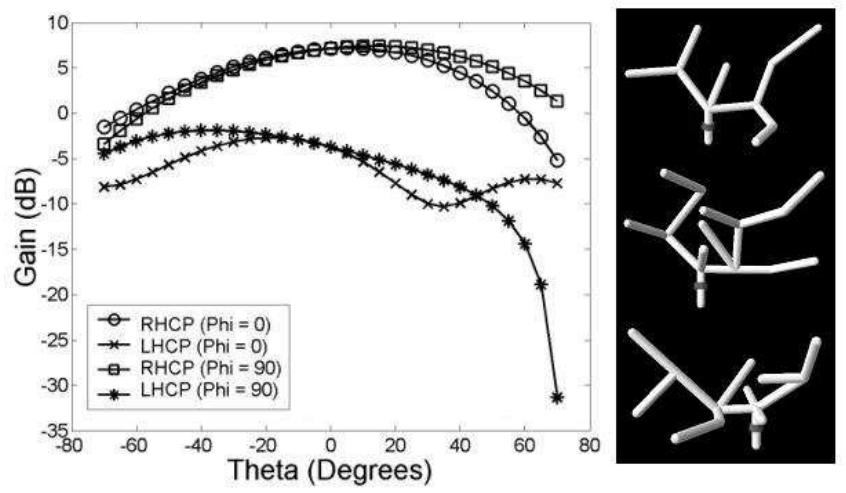

Fig. 1. Performance and sample designs.

The performance of Fig. 1 is representative of what was obtained for $45 \mathrm{~min}$ of computation. The VSWR is 1.63 with RHCP greater than LHCP over the range of elevation angles. Several sample antennas are also shown in Fig. 1. The image of the ground plane is omitted. The results indicate that the evolution of 3D wire antennas using a growth process, yields designs that are competitive with standard approaches. Full details will be given in the long version paper.

\section{REFERENCES}

[1] E. E. Altshuler, "Design of a Vehicular Antenna for GPS/Iridium using a Genetic Algorithm," IEEE Trans. Antennas Propagat., Vol. 48, No. 6, pp. 968-971, June 2000 .

[2] S. Rengarajan and Y. Rahmat-Samii, "On the Cross-Polarization Characteristics of Crooked Wire Antennas Designed by GeneticAlgorithms," IEEE Antennas and Propagation Society International Symposium, Vol. 1, pp. 706-709, San Antonio, USA, June 16-21, 2002. 